\title{
A CLASSIFICATION OF PRIME-VALENT REGULAR CAYLEY MAPS ON ABELIAN, DIHEDRAL AND DICYCLIC GROUPS
}

\author{
Dongseok Kim, Young Soo Kwon, and Jaeun Lee
}

\begin{abstract}
A Cayley map is a 2-cell embedding of a Cayley graph into an orientable surface with the same local orientation induced by a cyclic permutation of generators at each vertex. In this paper, we provide classifications of prime-valent regular Cayley maps on abelian groups, dihedral groups and dicyclic groups. Consequently, we show that all prime-valent regular Cayley maps on dihedral groups are balanced and all prime-valent regular Cayley maps on abelian groups are either balanced or anti-balanced. Furthermore, we prove that there is no prime-valent regular Cayley map on any dicyclic group.
\end{abstract}

\section{Introduction}

In this paper, we only consider undirected finite connected graphs without loops and multiple edges. For a simple graph $\Gamma$, an $\operatorname{arc}$ of $\Gamma$ is an ordered pair $(x, y)$ of adjacent vertices of $\Gamma$. Thus, every edge of $\Gamma$ gives rise to a pair of opposite arcs. By $V(\Gamma), E(\Gamma), D(\Gamma)$ and Aut $(\Gamma)$, we denote the vertex set, the edge set, the arc set and the automorphism group of $\Gamma$, respectively. A graph $\Gamma$ is said to be vertex-transitive, edge-transitive and arc-transitive if Aut $(\Gamma)$ acts transitively on the vertex set, the edge set and the $\operatorname{arc}$ set of $\Gamma$, respectively. A graph $\Gamma$ is one-regular if Aut $(\Gamma)$ is arc-transitive and the stabilizer of each arc in Aut $(\Gamma)$ is trivial. We consider Aut $(\Gamma)$ as an acting group on $V(\Gamma), E(\Gamma)$ and $D(\Gamma)$ according to the context.

For a simple graph $\Gamma$ with arc set $D$, an embedding of $\Gamma$ or a map with the underlying graph $\Gamma$ is a triple $\mathcal{M}=(D ; R, L)$, where $R$ is a permutation of $D$ whose orbits coincide with the sets of arcs based at the same vertex and $L$ is an involution of $D$ whose orbits are the pairs of arcs induced by the same edge. The permutations $R$ and $L$ are called a rotation and an arc-reversing involution of $\mathcal{M}$, respectively. Let $\operatorname{Mon}(\mathcal{M})$ be the permutation group $\langle R, L\rangle$ generated by $R$ and $L$ and call it a monodromy group of $\mathcal{M}$. Then, $\operatorname{Mon}(\mathcal{M})$ acts transitively

Received July 9, 2008; Revised June 8, 2009.

2000 Mathematics Subject Classification. 05C10, 05C30.

Key words and phrases. Cayley map, regular embedding.

This research was supported by the Yeungnam University research grants in 2007. 
on $D$. Given two maps $\mathcal{M}_{1}=\left(D_{1} ; R_{1}, L_{1}\right)$ and $\mathcal{M}_{2}=\left(D_{2} ; R_{2}, L_{2}\right)$, a map isomorphism $\phi: \mathcal{M}_{1} \rightarrow \mathcal{M}_{2}$ is a graph isomorphism between underlying graphs such that $\phi R_{1}(x, y)=R_{2} \phi(x, y)$ for every arc $(x, y) \in D_{1}$. In particular, if $\mathcal{M}_{1}=\mathcal{M}_{2}=\mathcal{M}$, then $\phi$ is called a map automorphism on $\mathcal{M}$. It follows that the automorphism group $\operatorname{Aut}(\mathcal{M})$ of $\mathcal{M}$ acts semi-regularly on $D$. If the action of Aut $(\mathcal{M})$ on $D$ is regular, then the map itself is called regular. It was shown that a map $\mathcal{M}$ is regular if and only if the monodromy group of a map $\mathcal{M}$ acts regularly on $D[9]$.

Let $G$ be a group and let $X=X^{-1}$ be a unit-free set of $G$ such that $\langle X\rangle=G$. A Cayley graph $\Gamma=\operatorname{Cay}(G, X)$ is a graph with vertex set $G$ and two vertices $g$ and $h$ are adjacent if and only if $g^{-1} h \in X$. The set of left translations $\tilde{G}=\left\{L_{g} \mid g \in G\right\}$, defined by $L_{g}(x)=g x$ forms a vertex-regular subgroup of Aut $(\Gamma)$. A Cayley graph Cay $(G, X)$ is normal if the left regular representation $\tilde{G}$ is a normal subgroup of Aut $($ Cay $(G, X))$. Note that the arc set $D$ of the Cayley graph Cay $(G, X)$ is $\{(g, g x) \mid g \in G, x \in X\}$. Let $q$ be a cyclic permutation of $X$. Then, a Cayley map $C M(G, X, q)$ is the map $\mathcal{M}=(D ; R, L)$ with the rotation defined by $R(g, g x)=(g, g q(x))$ and the arc-reversing involution $L$ defined by $L(g, g x)=(g x, g)$, where $g \in G$ and $x \in X$. It is easy to see that for every $g \in G, L_{g} R=R L_{g}$, hence $\tilde{G}$ is a subgroup of Aut $(\mathcal{M})$ acting regularly on vertices. Furthermore, a Cayley map $\mathcal{M}=C M(G, X, q)$ is regular if and only if there exists an automorphism $\rho$ in the stabilizer $(\text { Aut }(\mathcal{M}))_{v}$ of a vertex $v$ cyclically permuting the $|X|$ arcs based at $v$. In this case, $\operatorname{Aut}(\mathcal{M})$ is a product of $\tilde{G}$ with a cyclic group $\langle\rho\rangle \cong \mathbb{Z}_{n}$, where $n=|X|$ (see $[4,5])$.

For an integer $t$, a Cayley map $\mathcal{M}=C M(G, X, q)$ is t-balanced if $q(x)^{-1}=$ $q^{t}\left(x^{-1}\right)$ for every $x \in X$. In particular, a Cayley map $\mathcal{M}$ is balanced if it is 1-balanced. A balanced Cayley map $\operatorname{CM}(G, X, q)$ is regular if and only if there exists a group automorphism $\psi$ of $G$ whose restriction on $X$ is equal to $q$ [12]. In this case, the group $\tilde{G}$ is a normal subgroup of $\operatorname{Aut}(\mathcal{M})$ and $\operatorname{Aut}(\mathcal{M})$ is a semidirect product of $\tilde{G}$ by $\langle\psi\rangle$. On the other hand, a Cayley map $\mathcal{M}$ is anti-balanced if $\mathcal{M}$ is (-1)-balanced [13]. For more general theory of Cayley maps and their automorphisms, the reader is referred to $[4,5]$.

For any positive integer $n, D_{n}=\left\langle a, b \mid a^{n}=b^{2}=(a b)^{2}=1\right\rangle$ is the dihedral group of order $2 n$ and $\operatorname{Dic}_{n}=\langle a, b| a^{2 n}=1, b^{2}=a^{n}$ and $\left.b^{-1} a b=a^{-1}\right\rangle$ is the dicyclic group of order $4 n$. It is known $[6,8]$ that their automorphism groups are

$$
\begin{aligned}
\operatorname{Aut}\left(D_{n}\right)= & \left\{\sigma_{i, j} \mid \sigma_{i, j}(a)=a^{i}, \sigma_{i, j}(b)=a^{j} b, i, j \in\{1,2, \ldots, n\}\right. \\
& \text { and }(i, n)=1\},
\end{aligned}
$$

and

$$
\begin{aligned}
\operatorname{Aut}\left(\operatorname{Dic}_{n}\right)= & \left\{\alpha_{i, j} \mid \alpha_{i, j}(a)=a^{i}, \alpha_{i, j}(b)=a^{j} b, i, j \in\{1,2, \ldots, 2 n\}\right. \\
& \text { and }(i, 2 n)=1\} .
\end{aligned}
$$


In [1], M. Conder et al. developed a general theory of $t$-balanced Cayley maps and classified regular anti-balanced Cayley maps on abelian groups. In [6] and [8], J. H. Kwak et al. classified regular $t$-balanced Cayley maps on dihedral groups and dicyclic groups. This paper focuses on the classification of primevalent regular Cayley maps on abelian groups, dihedral groups and dicyclic groups. The main results are stated as the following three theorems.

Theorem 1.1. For any prime $p$, let $\mathcal{M}=C M(G, X, q)$ be a p-valent regular Cayley maps on abelian group $G$. Then $\mathcal{M}$ is either balanced or anti-balanced. Moreover,

(1) if $\mathcal{M}$ is balanced, then $\mathcal{M}$ is isomorphic to $C M\left(\mathbb{Z}_{2}^{n}, X_{1}, q_{1}\right)$ for some elementary abelian 2-group $\mathbb{Z}_{2}^{n}, X_{1} \subset \mathbb{Z}_{2}^{n}$ and a cyclic permutation $q_{1}$ of $X_{1}$ such that

(i) $X_{1}=\left\{A^{i} \mathbf{x} \mid 0 \leq i \leq p-1\right\}$ for some $A \in \mathrm{GL}_{n}(2)$ and $\mathbf{x} \in \mathbb{Z}_{2}^{n}$ satisfying that $p$ is the smallest positive integer such that $A^{p}=I$ and $\left\langle\mathbf{x}, A \mathbf{x}, A^{2} \mathbf{x}, \ldots, A^{p-1} \mathbf{x}\right\rangle=\mathbb{Z}_{2}^{n}$,

(ii) $q_{1}\left(A^{i} \mathbf{x}\right)=A^{i+1} \mathbf{x}$ for any $i \in\{0,1, \ldots, p-1\}$.

(2) If $\mathcal{M}$ is anti-balanced, then $\mathcal{M}$ is isomorphic to

$$
C M\left(\mathbb{Z}_{2 p}, X_{2}=\{1,3, \ldots, 2 p-1\}, q_{2}=\left(\begin{array}{lll}
1 & 3 & \cdots
\end{array} 2 p-1\right)\right) .
$$

Theorem 1.2. For any prime $p$, any p-valent regular Cayley map $\mathcal{M}=$ $C M\left(D_{n}, X, q\right)$ on a dihedral group is balanced. Furthermore, $\mathcal{M}$ is isomorphic to

$$
\begin{aligned}
C M\left(D_{n}, X_{1}=\right. & \left\{a^{\sum_{j=0}^{t} \ell^{j}} b \mid 0 \leq t \leq p-1\right\} \\
& \left.q_{1}=\left(b a b a^{\ell+1} b \cdots a^{\ell^{p-2}+\ell^{p-3}+\cdots+1} b\right)\right)
\end{aligned}
$$

for some positive integer $\ell<n$ such that $p$ is the smallest positive integer satisfying that $\ell^{p-1}+\ell^{p-2}+\cdots+1 \equiv 0(\bmod n)$.

Theorem 1.3. For any prime $p$, there is no p-valent regular Cayley map on a dicyclic group.

Our paper is organized as follows. In Section 2, we review some known results on maps, Cayley maps and transitive permutation groups of prime degree. In Section 3, the classifications of prime-valent regular Cayley maps on abelian groups, dihedral groups and on dicyclic groups are given. Furthermore, we give some remark that for fixed prime $p$, the underlying Cayley graphs of $p$-valent regular Cayley maps on dihedral groups are one-regular with finitely many exceptions.

\section{Preliminaries}

In this section, we review some results needed in the proofs of our main theorems.

For a $k$-valent Cayley map $\mathcal{M}=C M\left(\Gamma, X, q=\left(x_{1} x_{2} \cdots x_{k}\right)\right)$, let $\kappa$ be the involution on the set $\{1,2, \ldots, k\}$ such that for any $i=1,2, \ldots, k, x_{i}^{-1}=x_{\kappa(i)}$ 
and call it the distribution of inverses of $\mathcal{M}$. We denote the group generated by two permutations $(12 \cdots k)$ and $\kappa$ by $H(\kappa)$. For any $i=1,2, \ldots, k$, we denote the stabilizer of $i$ under $H(\kappa)$-action by $H(\kappa)_{i}$.

Proposition 2.1 ([10]). Let $\mathcal{M}=C M\left(G, X, q=\left(x_{1} x_{2} \cdots x_{k}\right)\right)=(D ; R, L)$ be a $k$-valent Cayley map with the distribution of inverses $\kappa$. Then, there exists a group epimorphism $f: \operatorname{Mon}(\mathcal{M}) \rightarrow H(\kappa)$ such that $f(R)=\left(\begin{array}{llll}1 & 2 & \cdots & k\end{array}\right)$, $f(L)=\kappa$ and $G$ is isomorphic to $f^{-1}\left(H(\kappa)_{i}\right) / \operatorname{Mon}(\mathcal{M})_{e}$ for some arc $e \in D$ and for some $i \in\{1,2, \ldots, k\}$ satisfying $f\left(\operatorname{Mon}(\mathcal{M})_{e}\right) \leq H(\kappa)_{i}$.

Proposition 2.1 implies that if $\mathcal{M}=C M\left(G, X, q=\left(x_{1} x_{2} \cdots x_{k}\right)\right)=(D$; $R, L)$ is a regular $k$-valent Cayley map with the distribution of inverses $\kappa$, then there exists a group epimorphism $f: \operatorname{Mon}(\mathcal{M}) \rightarrow H(\kappa)$ such that $G$ is isomorphic to $f^{-1}\left(H(\kappa)_{i}\right)$ for some $i, 1 \leq i \leq k$. It means that there is an epimorphism $g: G \rightarrow H(\kappa)_{i}$. Note that for any $i, j, 1 \leq i, j \leq k$, two stabilizers $H(\kappa)_{i}$ and $H(\kappa)_{j}$ are isomorphic because the group $H(\kappa)$ acts transitively on the set $\{1,2, \ldots, k\}$.

The following proposition gives a classification of transitive permutation groups of prime degree.

Proposition $2.2([2])$. Let $G$ be a transitive permutation group of prime degree p. Then, $G$ is isomorphic to one of the followings:

(i) the symmetric group $S_{p}$ or the alternating group $A_{p}$;

(ii) a subgroup of $\mathrm{AGL}_{1}(p)$, 1-dimensional affine group over the field $F$ of order $p$

(iii) a permutation representation of $\mathrm{PSL}_{2}(11)$ of degree 11 ;

(iv) one of the Mathiew groups $M_{11}$ or $M_{23}$ of degree 11 or 23, respectively;

(v) a projective group $H$ with $\mathrm{PSL}_{d}(q) \leq H \leq \mathrm{P}_{d}(q)$ of degree $p=\frac{q^{d}-1}{q-1}$.

Proposition 2.3 ([3]). For any positive integer $d>1$ and for any prime power $q$, the projective special linear group $\mathrm{PSL}_{d}(q)$ is simple except for $\mathrm{PSL}_{2}(2)$ and

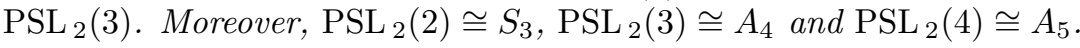

J. Širáň and M. Škoviera characterized regular balanced Cayley maps as follows.

Theorem 2.4 ([12]). A Cayley map $\mathcal{M}=C M(G, X, q)$ is a regular balanced if and only if there exists an automorphism $\rho$ of the group $G$ whose restriction on $X$ is equal to $q$.

M. Conder et al. classified regular anti-balanced Cayley maps on abelian groups. The classification implies the following result.

Proposition 2.5 ([1]). For a fixed prime $p \geq 3$, let $\mathcal{M}=C M(G, X, q)$ be a p-valent regular anti-balanced Cayley map on abelian group. Then, $\mathcal{M}$ is isomorphic to the Cayley map $C M\left(\mathbb{Z}_{2 p}, X_{1}, q_{1}\right)$, where

$$
X_{1}=\{1,3, \ldots, 2 p-1\} \quad \text { and } q_{1}=(13 \cdots 2 p-1) .
$$


Note that the underlying graph in Proposition 2.5 is the complete bipartite graph $K_{p, p}$.

In [6] and [8], J. H. Kwak et al. classified regular t-balanced Cayley maps on dihedral groups and dicyclic groups as follows.

Theorem 2.6 $([6])$. Let $\mathcal{M}=C M\left(D_{n}, X, q\right)$ be a regular anti-balanced Cayley map on a dihedral group with $|X| \geq 3$. Then, $n$ is even number $2 n^{\prime},|X|=4$ and $\mathcal{M}$ is isomorphic to a Cayley map $C M\left(D_{n}, X=\left\{b, a, a^{2 k} b, a^{-1}\right\}, q=\right.$ $\left.\left(b a a^{2 k} b a^{-1}\right)\right)$ for some $k$ satisfying $k^{2} \equiv-1\left(\bmod n^{\prime}\right)$.

Theorem $2.7([8])$. Let $\mathcal{M}=C M\left(\operatorname{Dic}_{n}, X, q\right)$ be a regular t-balanced Cayley map on a dicyclic group with $|X| \geq 3$. Then, $t=1$, i.e., $\mathcal{M}$ is balanced, and $\mathcal{M}$ is isomorphic to a Cayley map $C M\left(\operatorname{Dic}_{n}, X, q\right)$ with the cyclic permutation

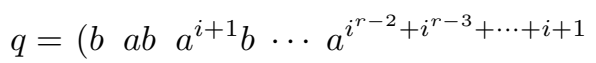

$$
\begin{aligned}
& \left.a^{n} b \quad a^{n+1} b \quad a^{n+i+1} b \quad \cdots \quad a^{n+i^{r-2}+i^{r-3}+\cdots+i+1}\right)
\end{aligned}
$$

on $X$, where $1 \leq i \leq 2 n-1,(i, 2 n)=1, r \geq 2$ and $i^{r-1}+i^{r-2}+\cdots+i+1 \equiv n$ $(\bmod 2 n)$.

For any positive integers $n, k$ and $\ell<n$ such that $k$ is the smallest positive integer satisfying $\ell^{k-1}+\ell^{k-2}+\cdots+1 \equiv 0(\bmod n)$, the Cayley map

$$
\begin{aligned}
C M\left(D_{n}, X=\right. & \left\{b, a b, a^{\ell+1} b, \ldots, a^{\ell^{k-2}+\ell^{k-3}+\cdots+1} b\right\} \\
& q=\left(\begin{array}{lllll}
b & a b & a^{\ell+1} b & \cdots & a^{\ell^{k-2}+\ell^{k-3}+\cdots+1} b
\end{array}\right)
\end{aligned}
$$

is known to be a regular balanced Cayley map [14]. For our convenience, let $\mathcal{T}$ be the set of all triples $(n, \ell, k)$ of positive integers such that $\ell<n$ and $k$ is the smallest integer satisfying $\ell^{k-1}+\ell^{k-2}+\cdots+1 \equiv 0(\bmod n)$. We denote the above Cayley map by $C M(n, \ell, k)$. Then, the following theorem holds.

Theorem 2.8 ([14]). Let $\mathcal{M}=C M\left(D_{n}, X, q\right)$ be a $k$-valent regular balanced Cayley map. Then, the map $\mathcal{M}$ is isomorphic to a Cayley map $C M(n, \ell, k)$, namely,

$$
\begin{aligned}
& C M\left(D_{n}, X=\left\{b, a b, a^{\ell+1} b, \ldots, a^{\ell^{k-2}+\ell^{k-3}+\cdots+1} b\right\},\right.
\end{aligned}
$$

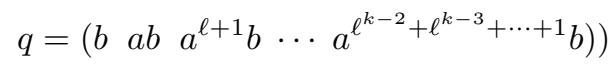

for some triple $(n, \ell, k) \in \mathcal{T}$. Moreover, for any two triples $\left(n, \ell_{1}, k\right),\left(n, \ell_{2}, k\right) \in$ $\mathcal{T}$, the regular balanced Cayley maps $C M\left(n, \ell_{1}, k\right)$ and $C M\left(n, \ell_{2}, k\right)$ are isomorphic if and only if $\ell_{1}=\ell_{2}$.

Theorem 2.8 implies that for any fixed integer $k \geq 3,\{C M(n, \ell, k) \mid(n, \ell, k)$ $\in \mathcal{T}\}$ is the set of all $k$-valent regular balanced Cayley maps on the dihedral groups up to isomorphisms. 


\section{Classifications of prime-valent Cayley maps}

In this section, we classify prime-valent regular Cayley maps on abelian groups, dihedral groups and dicyclic groups, respectively. First, we prove the following lemmas.

Lemma 3.1. For an odd prime $p$, let $G$ be a permutation group of degree $p$ generated by $\rho=\left(\begin{array}{lllll}1 & 2 & 3 & \cdots & p\end{array}\right)$ and $\kappa$, where $\kappa(p)=p$ and $\kappa^{2}$ is the identity. If $G$ is isomorphic to a subgroup of $\mathrm{AGL}_{1}(p)$, then $\kappa$ is either the identity or $\kappa=(p)(1 p-1)(2 p-2) \cdots\left(\frac{p-1}{2} \frac{p+1}{2}\right)$.

Proof. Because $\mathrm{AGL}_{1}(p)$ is a Frobenius group, $\langle\rho\rangle$ is a normal subgroup of $G$. Suppose that $\kappa(1)=i$. Then, $\kappa \rho \kappa \in\langle\rho\rangle$ and $\kappa \rho \kappa(p)=i$, which means $\kappa \rho \kappa=\rho^{i}$. It implies that for any $k=1,2, \ldots, p$,

$$
\kappa(k+i)=\kappa \rho^{i}(k)=\rho \kappa(k)=\kappa(k)+1 .
$$

If $i=1$, then $\kappa$ is the identity. Assume that $i \neq 1$. Inserting $k=i$, one can get $\kappa(2 i)=2$. And, taking $k=2 i$, one obtains $\kappa(3 i)=3$. By the same process, we find $\kappa(k i)=k$ for any $k=1,2, \ldots, p$. So, $\kappa\left(i^{2}\right)=i$. Since $\kappa(1)=i, i^{2} \equiv 1$ $(\bmod p)$. Therefore, $i=p-1$ and $\kappa=(p)(1 p-1)(2 p-2) \cdots\left(\frac{p-1}{2} \frac{p+1}{2}\right)$.

For an odd prime $p$ and a $p$-valent Cayley map $\mathcal{M}=C M(G, X, q)$ with the distribution of inverses $\kappa, \kappa$ fixes at least one element in $\{1,2, \ldots, p\}$. From now on, we assume that $\kappa(p)=p$ without loss of generality.

The next lemma shows that any prime-valent regular Cayley maps on abelian groups, dihedral groups or dicyclic groups are either balanced or anti-balanced.

Lemma 3.2. For an odd prime $p \geq 3$, let $\mathcal{M}=C M(G, X, q)$ be a p-valent regular Cayley map on an abelian group, a dihedral group or a dicyclic group with the distribution of inverses $\kappa$. Then, $\kappa$ is either the identity or $(p)(1 p-$ 1) $(2 p-2) \cdots\left(\frac{p-1}{2} \frac{p+1}{2}\right)$.

Proof. By Proposition 2.1, there exists a group epimorphism $f: \operatorname{Mon}(\mathcal{M}) \rightarrow$ $H(\kappa)$ such that $f(R)=\left(\begin{array}{llll}1 & 2 & \cdots & p\end{array}\right), f(L)=\kappa$ and $G$ is isomorphic to $f^{-1}\left(H(\kappa)_{i}\right)$ for some $i=1,2, \ldots, p$. It implies that the group $H(\kappa)_{i}$ is an epimorphic image of $G$. Since $G$ is an abelian group, a dihedral group or a dicyclic group, the group $H(\kappa)_{i}$ is either an abelian group, a dihedral group or a dicyclic group. Moreover, since the group $H(\kappa)$ generated by $(12 \cdots p)$ and $\kappa$ acts transitively on the set $\{1,2, \ldots, p\}, H(\kappa)$ is isomorphic to one of the groups in Proposition 2.2.

Case 1: $H(\kappa)$ is isomorphic to $S_{p}$ or $A_{p}$.

If $p=3$ and $H(\kappa) \cong S_{3}$, then $\kappa=(3)(12)$. If $p=3$ and $H(\kappa) \cong A_{3}$, then $\kappa$ is the identity.

Assume that $p>3$ and $H(\kappa)$ is isomorphic to $S_{p}$ or $A_{p}$. Then, for any $i=1,2, \ldots, p$, the stabilizer $H(\kappa)_{i}$ is isomorphic to neither an abelian group, a dihedral group nor a dicyclic group. Thus, it is impossible. 
Case 2: $H(\kappa)$ is isomorphic to a subgroup of $\mathrm{AGL}_{1}(p)$.

By Lemma 3.1, $\kappa$ is either the identity or $(p)(1 p-1)(2 p-2) \cdots\left(\frac{p-1}{2} \frac{p+1}{2}\right)$.

Case 3: $H(\kappa)$ is isomorphic to a permutation representation of $\mathrm{PSL}_{2}(11)$ of degree 11.

Because a permutation representation of $\mathrm{PSL}_{2}(11)$ of degree 11 is a transitive extension of a permutation representation of $A_{5}$ of degree $10, H(\kappa)_{i}$ should be isomorphic to $A_{5}$ for any $i=1,2, \ldots, 11$, which is isomorphic to neither an abelian group, a dihedral group nor a dicyclic group.

Case 4: $H(\kappa)$ is isomorphic to the Mathiew groups $M_{11}$ or $M_{23}$ of degree 11 or 23 , respectively.

Since both the Mathiew groups $M_{11}$ of degree 11 and $M_{23}$ of 23 act 4 transitively on 11-set and 23-set, respectively, the stabilizer $H(\kappa)_{i}$ acts 3transitively for any $i=1,2, \ldots, p$. Thus, there exist $\alpha, \beta \in H(\kappa)_{i}$ such that $\alpha=\left(\begin{array}{lll}x & y & z\end{array}\right) \cdots$ and $\beta=\left(\begin{array}{lll}y & z & w\end{array}\right) \cdots$ with $x \neq w$. Note that the orders of $\alpha$ and $\beta$ are multiples of 3. If $H(\kappa)_{i}$ is isomorphic to a dihedral group or a dicyclic group, then $\alpha$ and $\beta$ should commute. But, one can easily check $\alpha \beta \neq \beta \alpha$. Hence, $H(\kappa)_{i}$ can not be isomorphic to an abelian group, a dihedral group nor a dicyclic group.

Case 5: $H(\kappa)$ is isomorphic to a projective group $H$ with $\operatorname{PSL}_{d}\left(q_{1}\right) \leq H \leq$ $\mathrm{P \Gamma L}_{d}\left(q_{1}\right)$ of degree $p=\frac{q_{1}^{d}-1}{q_{1}-1}$ for some prime power $q_{1}$.

Subcase 5.1: $d>2$. The stabilizer of the point $[0,0, \ldots, 0,1]^{t}$ in $H$ contains a subgroup $S$ which is isomorphic to $\operatorname{PSL}_{d-1}\left(q_{1}\right)$. Except for $\left(d, q_{1}\right)=(3,2)$ or $(3,3)$, the group PSL $\operatorname{PL}_{-1}\left(q_{1}\right)$ is simple by Proposition 2.3. For $d=3$ and $q_{1}=3$, the group $\mathrm{PSL}_{2}(3)$ is isomorphic to the alternating group $A_{4}$. For $d=3$ and $q_{1}=2$, let

$$
A=\left[\begin{array}{lll}
1 & 1 & 0 \\
1 & 0 & 0 \\
1 & 1 & 1
\end{array}\right] \quad \text { and } \quad B=\left[\begin{array}{lll}
0 & 1 & 0 \\
1 & 1 & 0 \\
1 & 1 & 1
\end{array}\right]
$$

Then, $A$ and $B$ are in the stabilizer of the point $[0,0,1]^{t}$ in $\operatorname{PSL}_{3}(2)$ and the orders of $A$ and $B$ are 3 . Note that two elements of order 3 in a dihedral group or a dicyclic group commute. On the other hand, one can easily check $A B \neq B A$. Hence, for any $i=1,2, \ldots, p$, the stabilizer $H(\kappa)_{i}$ can not be isomorphic to an abelian group, a dihedral group nor a dicyclic group.

Subcase 5.2: $d=2$ and $q_{1}$ is an odd prime power. Then, the number $\frac{q_{1}^{2}-1}{q_{1}-1}=q_{1}+1$ can not be prime.

Subcase 5.3: $d=2$ and $q_{1}$ is $2^{r}$ for some positive integer $r$. If $q_{1}=2$, then $|X|=3$. It means that $\kappa$ is the identity or $(3)(12)$. Now, we assume that $q_{1}$ is $2^{r}$ for some positive integer $r>1$. Then, there exist non-identity elements $x, y \in G F\left(2^{r}\right)$ whose orders are odd, where $G F\left(2^{r}\right)$ is the Galois field of order 
$2^{r}$. For these elements $x, y \in G F\left(2^{r}\right)$, let

$$
C=\left[\begin{array}{ll}
1 & 0 \\
0 & x
\end{array}\right] \text { and } E=\left[\begin{array}{ll}
1 & y \\
0 & x
\end{array}\right] .
$$

Then, $C$ and $E$ are in the stabilizer of the point $[1,0]^{t}$ in $\operatorname{PSL}_{2}\left(2^{r}\right)$ and the order of $C$ is equal to that of $x$, hence it is odd. Note that two elements of odd order in a dihedral group or a dicyclic group should commute. One can easily check $C E \neq E C$. Thus, the stabilizer of the point $[1,0]^{t}$ in $\operatorname{PSL}_{2}\left(2^{r}\right)$ is isomorphic to neither an abelian group, a dihedral group nor a dicyclic group.

Therefore, in all cases, $\kappa$ is either the identity or

$$
(p)(1 p-1)(2 p-2) \cdots\left(\frac{p-1}{2} \frac{p+1}{2}\right) .
$$

Note that if the distribution of inverses $\kappa$ of a Cayley map $\mathcal{M}=C M(G, X, q)$ is the identity, then $\mathcal{M}$ is balanced and all elements (generators) in $X$ are involutions. On the other hand, if $\kappa$ is $(p)(1 p-1)(2 p-2) \cdots\left(\frac{p-1}{2} \frac{p+1}{2}\right)$, then the map $\mathcal{M}$ is anti-balanced.

Proof of Theorem 1.1. Let $\mathcal{M}=C M(G, X, q)$ be a $p$-valent regular Cayley map on an abelian group $G$ with the distribution of inverses $\kappa$. Then, by Lemma $3.2, \kappa$ is either the identity or $(p)(1 p-1)(2 p-2) \cdots\left(\frac{p-1}{2} \frac{p+1}{2}\right)$.

Assume that $\kappa$ is the identity. Then, $\mathcal{M}$ is balanced and all elements in $X$ are involutions. Furthermore, $X$ is an orbit under an automorphism $\psi$ of $G$ and the restriction of $\psi$ on $X$ is $q$. Since $G$ is an abelian group and $G$ is generated by involutions, $G$ is isomorphic to an elementary abelian 2-group $\mathbb{Z}_{2}^{n}$ for some positive integer $n$. Note that the automorphism group of $\mathbb{Z}_{2}^{n}$ is $\mathrm{GL}_{n}(2)$. Hence, $\mathcal{M}$ is isomorphic to $C M\left(\mathbb{Z}_{2}^{n}, X_{1}, q_{1}\right)$ such that

(i) $X_{1}=\left\{A^{i} \mathbf{x} \mid 0 \leq i \leq p-1\right\}$ for some $A \in \mathrm{GL}_{n}(2)$ and $\mathbf{x} \in \mathbb{Z}_{2}^{n}$ satisfying that $p$ is the smallest positive integer such that $A^{p}=I$ and $\left\langle\mathbf{x}, A \mathbf{x}, A^{2} \mathbf{x}, \ldots, A^{p-1} \mathbf{x}\right\rangle=\mathbb{Z}_{2}^{n}$ and

(ii) $q_{1}\left(A^{i} \mathbf{x}\right)=A^{i+1} \mathbf{x}$ for any $i \in\{0,1, \ldots, p-1\}$.

Next, we assume that $\kappa$ is $(p)(1 p-1)(2 p-2) \cdots\left(\frac{p-1}{2} \frac{p+1}{2}\right)$. Then, $\mathcal{M}$ is anti-balanced. By Proposition 2.5, $\mathcal{M}$ is isomorphic to

$$
C M\left(\mathbb{Z}_{2 p}, X_{2}=\{1,3, \ldots, 2 p-1\}, q_{2}=\left(\begin{array}{lll}
1 & 3 \cdots 2 p-1)
\end{array}\right) .\right.
$$

Next, we proceed to prove Theorems 1.2 and 1.3 .

Proof of Theorem 1.2. Let $\mathcal{M}=C M\left(D_{n}, X, q\right)$ be a $p$-valent regular Cayley map on dihedral group with the distribution of inverses $\kappa$. Then, by Lemma 3.2, $\kappa$ is either the identity or $(p)(1 p-1)(2 p-2) \cdots\left(\frac{p-1}{2} \frac{p+1}{2}\right)$.

Suppose that $\kappa$ is $(p)(1 p-1)(2 p-2) \cdots\left(\frac{p-1}{2} \frac{p+1}{2}\right)$. Then, $\mathcal{M}$ is antibalanced. By Theorem 2.6, all regular anti-balanced Cayley maps on dihedral groups are 4 -valent. So, it is impossible. Therefore, $\kappa$ is the identity and the map $\mathcal{M}$ is balanced. By Theorem 2.8, it completes the proof. 
Corollary 3.3. For any prime $p$, let $\Gamma=\operatorname{Cay}\left(D_{n}, X\right)$ be a $p$-valent one-regular Cayley graph on dihedral group. Then, $\Gamma$ is normal.

Proof. For the identity element 1 in $D_{n}$, the stabilizer Aut $(\Gamma)_{1}$ is a cyclic group of order $p$ and acts regularly on the neighborhood $N(1)$ of 1 . Hence, there is a regular Cayley map $\mathcal{M}=C M\left(D_{n}, X, q\right)$ whose underlying graph is $\Gamma=$ Cay $\left(D_{n}, X\right)$. By Theorem $1.2, \mathcal{M}$ is balanced. Therefore, the left translation subgroup $\tilde{D_{n}}$ is a normal subgroup of $\operatorname{Aut}(\Gamma)=\operatorname{Aut}(\mathcal{M})$.

Proof of Theorem 1.3. Let $\mathcal{M}=C M\left(\operatorname{Dic}_{n}, X, q\right)$ be a $p$-valent regular Cayley map on a dicyclic group with the distribution of inverses $\kappa$. Then, by Lemma 3.2, $\mathcal{M}$ is either balanced or anti-balanced. By Theorem 2.7, there is no regular anti-balanced Cayley maps on dicyclic groups and every valancy of regular balanced Cayley maps on dicyclic groups is even. Therefore, there is no prime-valent regular Cayley map on dicyclic groups.

Remark 1. For any prime $p$, any $p$-valent one-regular Cayley graphs $\Gamma=$ Cay $\left(D_{n}, X\right)$ on dihedral groups are underlying graphs of regular balanced Cayley maps on dihedral groups by Theorem 1.2 and Corollary 3.3. So, $\Gamma$ is isomorphic to Cay $\left(D_{n}, X=\left\{b, a b, a^{\ell+1} b, \ldots, a^{\ell^{p-2}+\ell^{p-3}+\cdots+1} b\right\}\right)$ for some $\ell$ such that $0<\ell<n$ and $\ell^{p-1}+\ell^{p-2}+\cdots+1 \equiv 0(\bmod n)$. In [7], it is shown that for any prime $p$ and for any $n, \ell$ satisfying that $p$ is the smallest positive integer such that $\ell^{p-1}+\ell^{p-2}+\cdots+\ell+1 \equiv 0(\bmod n)$, the Cayley graph Cay $\left(D_{n}, X=\{b\right.$, $\left.\left.a b, a^{\ell+1} b, \ldots, a^{\ell^{p-2}+\ell^{p-3}+\cdots+1} b\right\}\right)$ is one-regular except for finitely many such pairs $n$ and $\ell$. Namely, there exists a constant $M$ which depends on $p$ such that for any $n>M$ and $(n, \ell, p) \in \mathcal{T}$, the Cayley graph Cay $\left(D_{n}, X=\{b, a b\right.$, $\left.\left.a^{\ell+1} b, \ldots, a^{\ell^{k-2}+\ell^{k-3}+\cdots+1} b\right\}\right)$ is one-regular. In [7], it is also shown that for any $n \geq 31$ and $(n, \ell, 5) \in \mathcal{T}$, the Cayley graph

$$
\text { Cay }\left(D_{n}, X=\left\{b, a b, a^{\ell+1} b, a^{\ell^{2}+\ell+1} b, a^{\ell^{3}+\ell^{2}+\ell+1} b\right\}\right)
$$

is one-regular.

By Theorems 2.8 and 1.2, for a given positive integer $n$ and for a given prime $p$, the number of non-isomorphic $p$-valent regular Cayley maps on $D_{n}$ is the number of positive integer $\ell$ satisfying $(n, \ell, p) \in \mathcal{T}$. Note that if $\ell^{p-1}+$ $\ell^{p-2}+\cdots+1 \equiv 0(\bmod n)$, then $(\ell-1)\left(\ell^{p-1}+\ell^{p-2}+\cdots+1\right)=\ell^{p}-1 \equiv 0$ $(\bmod n)$. So, the numbers $n$ and $\ell$ are relatively prime. For any positive integer $n=2^{a} p_{1}^{a_{1}} p_{2}^{a_{2}} \cdots p_{t}^{a_{t}}\left(p_{1}, p_{2}, \ldots, p_{t}\right.$ are distinct odd prime numbers, $a \geq 0$ and $a_{i}>0$ for each $i \geq 1$ ), the multiplicative group $\mathbb{Z}_{n}^{*}$ is isomorphic to the product of multiplicative groups $\mathbb{Z}_{2^{a}}^{*} \times \mathbb{Z}_{p_{1}^{a_{1}}}^{*} \times \cdots \times \mathbb{Z}_{p_{t}}^{*}$ by Chinese Remainder Theorem. Moreover, it is well known that $\mathbb{Z}_{2}^{*}=\{1\}, \mathbb{Z}_{4}^{*} \cong \mathbb{Z}_{2}, \mathbb{Z}_{2^{a}}^{*} \cong \mathbb{Z}_{2} \times \mathbb{Z}_{2^{a-2}}$ for $a \geq 3$ and $\mathbb{Z}_{p_{i}^{a_{i}}}^{*} \cong \mathbb{Z}_{p_{i}^{a^{i}-1}\left(p_{i}-1\right)}$ for any odd prime $p_{i}$ with $a_{i} \geq 1$ [11].

Theorem 3.4. Let $p$ be an odd prime and let $n=p_{0}^{a_{0}} p_{1}^{a_{1}} p_{2}^{a_{2}} \cdots p_{t}^{a_{t}}\left(p_{0}, p_{1}, p_{2}\right.$, $\ldots, p_{t}$ are distinct primes and $p_{0}=p, a_{0} \geq 0$ and $a_{i}>0$ for each $\left.i \geq 1\right)$ be $a$ 
positive integer. Then, the number of non-isomorphic p-valent regular Cayley maps on the dihedral group $D_{n}$ is $(p-1)^{t}$ if $a_{0}=0$ or 1 and $p \mid\left(p_{i}-1\right)$ for all $i=1,2, \ldots, t ; 0$ otherwise.

Proof. Let $\ell$ be a positive integer such that $\ell<n$ and $\ell^{p-1}+\ell^{p-2}+\cdots+1 \equiv 0$ $(\bmod n)$. Then, the numbers $n$ and $\ell$ are relatively prime and $\ell^{p-1}+\ell^{p-2}+\cdots+$ $1 \equiv 0\left(\bmod p_{i}^{a_{i}}\right)$ for any $i=0,1, \ldots, t$. Assume that $\ell \equiv \ell_{i}\left(\bmod p_{i}^{a^{i}}\right)$ with $0<\ell_{i}<p_{i}^{a_{i}}$ for any $i=0,1, \ldots, t$. If $\ell_{i} \equiv 1\left(\bmod p_{i}\right)$ for some $i=1,2, \ldots, t$, then

$$
\ell^{p-1}+\ell^{p-2}+\cdots+1 \equiv \ell_{i}^{p-1}+\ell_{i}^{p-2}+\cdots+1 \equiv p \neq 0 \quad\left(\bmod p_{i}\right)
$$

which is a contradiction. Hence, for any $i=1,2, \ldots, t, \ell_{i} \neq 1\left(\bmod p_{i}\right)$. It implies that the equation $\ell^{p-1}+\ell^{p-2}+\cdots+1 \equiv 0\left(\bmod p_{i}^{a_{i}}\right)$ is equivalent to the equation $\ell_{i}^{p} \equiv 1\left(\bmod p_{i}^{a_{i}}\right)$ for $i=1,2, \ldots, t$. If there exists an $i \in\{1,2, \ldots, t\}$ such that $p \nmid\left(p_{i}-1\right)$, then there is no such $\ell_{i}$ because $\left|\mathbb{Z}_{p_{i} a_{i}}^{*}\right|=p_{i}^{a_{i}-1}\left(p_{i}-1\right)$. For any $p_{i}$ satisfying $p \mid\left(p_{i}-1\right)$, there exist $p-1$ elements $\ell_{i}$ such that $\ell_{i} \neq 1$ $\left(\bmod p_{i}\right)$ and $\ell_{i}^{p} \equiv 1\left(\bmod p_{i}^{a_{i}}\right)$.

Assume that $a_{0}=1$. Then, for any $x \in \mathbb{Z}_{p}-\{1\}, x^{p-1}+x^{p-2}+\cdots+1=$ $\frac{x^{p}-1}{x-1} \equiv 1(\bmod p)$. Thus, $\ell_{0}=1$ is the only integer such that $0<\ell_{0}<p$ and $\ell_{0}^{p-1}+\ell_{0}^{p-2}+\cdots+1 \equiv 0(\bmod p)$.

Suppose that $a_{0} \geq 2$. For any $x \in \mathbb{Z}_{p^{a_{0}}}$ with $x \neq 1(\bmod p), x^{p-1}+x^{p-2}+$ $\cdots+1=\frac{x^{p}-1}{x-1} \equiv 1(\bmod p)$. Hence, $\ell_{0}$ is $s p+1$ for some $s\left(0<s<p^{a_{0}-1}\right)$. Moreover, $\ell_{0}$ is $s^{\prime} p^{a_{0}-1}+1$ for some $s^{\prime}\left(0<s^{\prime}<p\right)$ because $\ell_{0}$ should satisfy $\ell_{0}^{p}=(s p+1)^{p} \equiv 1\left(\bmod p^{a_{0}}\right)$. But, for such a number $\ell_{0}$,

$$
\begin{aligned}
& \ell_{0}^{p-1}+\ell_{0}^{p-2}+\cdots+1 \\
\equiv & \left((p-1) s^{\prime} p^{a_{0}-1}+1\right)+\left((p-2) s^{\prime} p^{a_{0}-1}+1\right)+\cdots+\left(s^{\prime} p^{a_{0}-1}+1\right)+1 \\
\equiv & p\left(\bmod p^{a_{0}}\right) .
\end{aligned}
$$

Therefore, there exists no such an $\ell_{0}$.

Conversely, for any $\ell_{0}, \ell_{1}, \ldots, \ell_{t}$ such that $0<\ell_{i}<p_{i}^{a_{i}}$ and $\ell_{i}^{p-1}+\ell_{i}^{p-2}+$ $\cdots+1 \equiv 0\left(\bmod p_{i}^{a_{i}}\right)$ for any $i=0,1, \ldots, d$, there exists a unique $\ell$ such that $0<\ell<n, \ell \equiv \ell_{i}\left(\bmod p_{i}^{a_{i}}\right)$ and $\ell^{p-1}+\ell^{p-2}+\cdots+1 \equiv 0(\bmod n)$ by Chinese Remainder Theorem. Therefore, the number of $\ell$ 's satisfying $0<\ell<n$ and $\ell^{p-1}+\ell^{p-2}+\cdots+1 \equiv 0(\bmod n)$ is $(p-1)^{t}$ if $a_{0}=0$ or 1 and $p \mid\left(p_{i}-1\right)$ for all $i=1,2, \ldots, t ; 0$ otherwise.

\section{References}

[1] M. Conder, R. Jajcay, and T. Tucker, Regular t-balanced Cayley maps, J. Combin. Theory Ser. B 97 (2007), no. 3, 453-473.

[2] J. Dixon and B. Mortimer, Permutation Groups, Springer-Verlag, New York, 1996.

[3] L. C. Grove, Classical Groups and Geometric Algebra, American Mathematical Society, GSM 39, 2001.

[4] R. Jajcay, Automorphism groups of Cayley maps, J. Combin. Theory Ser. B 59 (1993), no. $2,297-310$ 
[5] R. Jajcay and J. Širáň, Skew-morphisms of regular Cayley maps, Discrete Math. 244 (2002), no. 1-3, 167-179.

[6] J. H. Kwak, Y. S. Kwon, and R. Feng, A classification of regulart-balanced Cayley maps on dihedral groups, European J. Combin. 27 (2006), no. 3, 382-393.

[7] J. H. Kwak, Y. S. Kwon, and J. M. Oh, Infinitely many one-regular Cayley graphs on dihedral groups of any prescribed valency, J. Combin. Theory Ser. B 98 (2008), no. 3, 585-598.

[8] J. H. Kwak and J.-M. Oh, A classification of regular t-balanced Cayley maps on dicyclic groups, European J. Combin. 29 (2008), no. 5, 1151-1159.

[9] R. Nedela, Regular maps - combinatorial objects relating different fields of mathematics, J. Korean Math. Soc. 38 (2001), no. 5, 1069-1105.

[10] B. Richter, J. Širáň, R. Jajcay, T. Tucker, and M. Watkins, Cayley maps, J. Combin. Theory Ser. B 95 (2005), no. 2, 189-245.

[11] J. J. Rotman, An Introduction to the Theory of Groups, Graduate Texts in Mathematics, 148. Springer-Verlag, New York, 1995.

[12] M. Škoviera and J. Širáň, Regular maps from Cayley graphs. I. Balanced Cayley maps, Discrete Math. 109 (1992), no. 1-3, 265-276.

[13] J. Širán̆ and M. Škoviera, Regular maps from Cayley graphs. II. Antibalanced Cayley maps, Discrete Math. 124 (1994), no. 1-3, 179-191.

[14] Y. Wang and R. Feng, Regular balanced Cayley maps for cyclic, dihedral and generalized quaternion groups, Acta Math. Sin. (Engl. Ser.) 21 (2005), no. 4, 773-778.

DONGSEOK KIM

Department of Mathematics

KYUNGGI UNIVERSITY

SuWON 442-760, KOREA

E-mail address: dongseok@knu.ac.kr

YOUNG SOO KWON

Department of Mathematics

YEUNGNAM UNIVERSITY

KYONGSAN 712-749, KOREA

E-mail address: ysookwon@ynu.ac.kr

JAEUN LEE

Department of Mathematics

YEUNGNAM UNIVERSITY

KYONGSAN 712-749, KorEA

E-mail address: julee@yu.ac.kr 